\title{
Estructuración y formulación de la política pública farmacéutica regional y territorial en Colombia*
}

\section{Structuring and Development of Regional and Territorial Pharmaceutical Public Policy in Colombia}

\section{Estruturação e formulação da política pública farmacêutica regional e territorial na Colômbia}

Fecha de recepción: 26-05-15 Fecha de aceptación: 18-08-15 Disponible en línea: 01-11-15 doi: 10.11144/Javeriana.rgyps14-29.efpp

Cómo citar este artículo:

Gutiérrez Ossa JA, Manrique Hernández RD. Estructuración y formulación de la política pública farmacéutica regional y territorial en Colombia. Rev. Gerenc. Polít. Salud. 2015; 14(29): 41-59. http://dx.doi.org/10.11144/Javeriana.rgyps14-29.efpp

Este artículo de reflexión es producto del proyecto de investigación Estructura de las Empresas y la Industria Químico Farmacéutica Frente a la Economía de la Salud y la Geopolítica de los Medicamentos: Una Revisión Internacional y Desarrollo en el Caso Colombiano. Código INV032013003, financiado por la Dirección de Investigaciones de la Universidad CES (Medellín) y la Universidad del Rosario (Bogotá), en el periodo de julio del 2013 a septiembre del 2014, llevado a cabo por el grupo de investigación en Gestión Empresarial de la Facultad de Administración CES, registrado en Colciencias en categoría D, el Grupo de Investigación en Perdurabilidad Empresarial (GIPE) de la Facultad de Administración de la Universidad del Rosario y el Grupo ICIF-CES de la Facultad de Química Farmacéutica de la Universidad CES, registrado en Colciencias.

** Economista de la Universidad de Medellín, Colombia, Ph.D. en Administración Pública de la Atlantic International University (AIU), Honolulú, Estados Unidos; magíster en Desarrollo de la Universidad Pontificia Bolivariana, Medellín, Colombia; investigador y líder del Grupo de Gestión Empresarial del CES, Facultad de Ciencias Administrativas, Universidad CES y Universidad del Rosario, Medellín, Colombia. Correo electrónico: algutierrez@ ces.edu.co; jagogutierrez@gmail.com, Tel: 4440555, Extensión; 1411, Dirección: Calle10 A No 22-04. Piso 4, Facultad Administración de Empresas, Medellín, Colombia.

*** Químico farmaceuta de la Universidad de Antioquia, Medellín, Colombia. Ph.D. (c) en Epidemiología, Universidad de Antioquia; magíster en Epidemiología de la Universidad de Antioquia; especialista en Altas Técnicas del Laboratorio Forense; investigador del Grupo de Investigación ICIF-CES; director de la Unidad de Gestión del Conocimiento de la Universidad CES en Medellín. Correo electrónico: rmanrique@ces.edu.co. Tel: 4440555, ext.: 1604, Calle 10를. No. 22- 6C- 103. Edificio administrativo Monticello, cuarto piso. Dirección de Gestión del Conocimiento, Universidad CES-Medellín, Antioquia 


\section{Resumen}

Problema: radica en la estructuración y formulación de la política pública farmacéutica regional y territorial en Colombia. Justificación: el documento Conpes 155 exige la definición del marco de política pública farmacéutica en Colombia, y por ende a escala departamental y territorial. Método: la fundamentación analítica de dicho Conpes, al igual que el uso de la metodología de marco lógico, fungen como herramientas metódicas para la postulación del trabajo. Marco de referencia: revisión en materia de definición u observancia de políticas públicas farmacéuticas internacionales. Principales resultados: el planteamiento de la respectiva política pública farmacéutica en el departamento de Antioquia y la postulación de dicho escenario en la ciudad capital de Medellín. Conclusiones: la construcción de la política pública farmacéutica nacional aún está en ciernes, obstáculo que impide registrarla en la política pública territorial.

Palabras clave: formulación de políticas; química farmacéutica; política nacional de medicamentos; política nacional de asistencia farmacéutica; política pública; políticas públicas de salud

\section{Abstract}

The problem lies on the structuring and development of regional and territorial public drug policy in Colombia. Justification: The document Conpes 155 requires the definition of the public pharmaceutical policy framework in Colombia, and therefore defining it at the departmental and local level. Method: the analytical foundation of the Conpes like the use of the logical framework methodology, serve as methodical tools for the application of the project. Framework: review of the definition or compliance of international drug policies. Main results: the approach of the public pharmaceutical policy in the department of Antioquia and the application of such a scenario in the capital city of Medellin. Conclusions: the construction of a public national drug policy is still in its infancy, fact that hinders the establishment of a territorial public policy.

Keywords: policy formulation; pharmaceutical chemistry; national drug policy; national pharmaceutical policy; public policy; public health policies

\section{Resumo}

Problema: reside na estruturação e formulação da política pública farmacêutica regional e territorial na Colômbia. Justificação: o documento Conpes 155 exige a definição do quadro de política pública farmacêutica na Colômbia, e, portanto, na escala departamental e territorial. Método: a fundamentação analítica de tal Conpes, mesmo que o uso da metodologia de quadro lógico, agem como ferramentas metódicas para a execução do trabalho. Quadro de referência: revisão em matéria de definição ou observância de políticas públicas farmacêuticas internacionais. Principais resultados: a colocação da respetiva política pública farmacêutica no departamento de Antioquia e a proposição de tal cenário na cidade capital de Medellín. Conclusões: a construção da política pública farmacêutica nacional ainda está na infância, obstáculo que impede cadastrá-la na política pública territorial.

Palavras-chave: formulação de política, química farmacêutica, política nacional de medicamentos, política nacional de assistência farmacêutica, política pública, políticas públicas de saúde 


\section{Introducción}

El interés de establecer las bases de la política farmacéutica nacional (PFN) en Colombia representa un ejercicio propositivo para el sistema de salud colombiano. La definición de la PFN exige el reconocimiento del modelo o sistema internacional de medicamentos. De paso, la definición del alcance metodológico sobre el cual estimar la pfn, para finalmente proponer los elementos requeridos para la política pública farmacéutica territorial (PFT).

La elaboración de las políticas públicas requiere el reconocimiento previo de la política económica y/o de la legislación establecida para el sector sobre el cual se pretende establecer o proponer dichas políticas. En general, la política pública emerge de las condiciones que caracterizan a los sectores, y no de otra manera, por ende, la PFN y la PFT están sujetas a las condiciones generales de la economía, del sistema de salud y del sector de medicamentos.

El componente metodológico corre por cuenta del análisis de marco lógico, iniciativa metodológica de carácter participativo sobre la que los distintos actores tienen convocatoria y participación. Con ello se pretende considerar los elementos que deben contemplarse, básicamente, para hacer factibles la PFN y la PFT. No obstante, es importante reconocer el proceso que dicha metodología implica a la hora de llevar el ejercicio por dicho marco.

La expectativa creada por los lineamientos del documento Conpes 155, y de paso por el interés de propugnar por un marco de política pública farmacéutica que atienda ambos frentes, lleva a postular los elementos que debe contener dicha iniciativa para cualquier departamento. Es preciso indicar que la política pública se construye a partir de actores interesados, pero igualmente a partir de las condiciones que permitan su sostenimiento.

En aras de analizar los elementos aquí planteados, el artículo obedece a los siguientes criterios: 1) estructuración y generalidades de la política pública farmacéutica nacional en Colombia; 2) análisis metodológico de la política pública farmacéutica nacional y de la política pública farmacéutica territorial; y 3) postulación de un ejercicio de política pública farmacéutica a escala regional y territorial. En suma, puntos que requieren revisión nacional.

\section{Estructuración y generalidades de la política pública farmacéutica nacional en Colombia}

La PFN atiende los acuerdos, pactos y tratados vinculantes dirigidos a fortalecer la base de medicamentos, conforme a las prioridades de cada país. En Colombia se ha dado paso para que se aborde la PFN de acuerdo con los preceptos establecidos en el documento Conpes 155 , cuya idea es definir la política general y, asimismo, para cada departamento y municipio del país. Por ende, la importancia de considerar los pormenores de dicha iniciativa y de su alcance:

Este documento presenta a consideración del Consejo Nacional de Política Económica y Social (Conpes) la Política Farmacéutica $(\mathrm{PF})$, formulada en el marco de los principios constitucionales asociados al derecho a la salud y de aquellos elementos de desarrollo del sector industrial farmacéutico que faciliten el cumplimiento de este derecho. La Política se encuentra articulada y responde a los objetivos planteados en el Plan Nacional de Desarrollo 2010-2014 "Prosperidad para Todos". (1, p. 3) 
El contenido de la política pública nacional corresponde a los preceptos que en materia constitucional, legislativa, normativa y de política económica, además de la política o estrategia de salud, se plantean en dicha materia u otras. No se trata de una política aislada de los agentes principales y de los subsectores que representan al sector de la salud y específicamente al subsector farmacéutico. Se trata de una política en la que caben las condiciones de mercado, pero también las iniciativas públicas y colectivas interesadas en intervenir en el tema:

Las listas de medicamentos esenciales a nivel institucional, local y nacional constituyen un elemento básico de las políticas de salud en general y de medicamentos en particular. (2, p. 184)

La política pública emerge cuando las condiciones en materia de política económica e institucional son propicias para elevar la figura a dicha naturaleza, entre las que tiene espacio la posibilidad de considerar a los medicamentos como esenciales para la vida. La política pública tiene un amplio sustento en pactos, en el que los diferentes agentes-principales, grupos de presión, intereses individuales y colectivos convergen para desarrollarla:

La Organización Mundial de la Salud y la Organización Panamericana de la Salud han venido otorgando apoyo a los diferentes estamentos gubernamentales con el objetivo primordial de colaborar en políticas sanitarias que beneficien a la población de todos los países. En esta oportunidad se desea entregar una herramienta que permita, a través de indicadores, conocer la situación farmacéutica de un país, establecer hasta donde han sido eficientes las políticas adoptadas en él y permitir formular estrategias 44 para solucionar las posibles fallas en su desarrollo y aplicación. (3, p. 7)
El conjunto de elementos sobre los cuales trasiega la política pública comprende tanto las pretensiones que en la materia exponen organismos multilaterales como la Organización Mundial de la Salud (oms) o la Organización Panamericana de la Salud (ops), marcos de referencia importantes a la hora de abonar las características y patrones que terminan por guiar a la PFN y a la PFT y, por ende, elevar dicha temática a interés público nacional:

En el campo internacional, ha tenido lugar un incremento de la aplicación de las evaluaciones fármaco-económicas para cuantificar el valor de los servicios y productos farmacéuticos en el contexto de los sistemas de salud, ya que correctamente utilizados aportan una información clave en el proceso de decisión. (4, p. 63)

La evidencia vinculada a la formulación sobre política farmacéutica internacional es exigua. En consecuencia, habría que sopesar los puntos de esta propuesta, en medio de un desbordado sistema de salud. Así que la disposición de la política farmacéutica nacional, en cualquier caso, tiende a generar postulados en la materia más que a priorizar un ajuste institucional, en donde cuesta hablar en propiedad de políticas públicas bajo dichas circunstancias.

La convocatoria de agentes-principales, pacientes y usuarios, industriales y grandes laboratorios, a la par que multinacionales y trasnacionales, representa el conjunto de actores sobre los cuales se cimenta la generación de la política pública. Es un trabajo que tiende a colectivizarse, a pesar del aparente estado de separación; además, para que la política tenga efectividad debe contar con la participación de todos los involucrados en ella:

Respaldar la aprobación del documento del Consejo Nacional de Política Económica 
Tabla 1. Principales factores Que influyen en el uSo de Medicamentos POR Parte de los consumidores

\begin{tabular}{|c|c|}
\hline NIVEL DE INFLUENCIA & FACTORES \\
\hline La familia & $\begin{array}{l}\text { - } \quad \text { Percepción de la necesidad de medicamentos } \\
\text { - } \quad \text { Ideas sobre eficacia e inocuidad Desconocimiento que lleva a la } \\
\text { - } \quad \text { Politerapia } \\
\text { - } \quad \text { Precio de los medicamentos } \\
\text { - } \quad \text { Nivel de alfabetización } \\
\text { - } \quad \text { "Poder" de los medicamentos }\end{array}$ \\
\hline La comunidad & $\begin{array}{l}\text { - } \quad \text { Patrones culturales de uso de medicamentos } \\
\text { - } \quad \text { Sistemas de suministro de medicamentos } \\
\text { - } \quad \text { Canales de información }\end{array}$ \\
\hline Las instituciones sanitarias & $\begin{array}{ll}\text { - } & \text { Frecuencia de la consulta a los agentes de salud } \\
\text { - } & \text { Calidad de la prescripción } \\
\text { - } & \text { Calidad de la consulta } \\
\text { - } & \text { Calidad de la dispensación } \\
\text { - } & \text { Suministro regular } \\
\text { - } & \text { Precio de los medicamentos }\end{array}$ \\
\hline El plano nacional & $\begin{array}{ll}\text { - } & \text { Aplicación de políticas de medicamentos esenciales } \\
\text { - } & \text { Promoción de medicamentos Financiamento y reembolsos } \\
\text { - } & \text { Defensa del consumidor } \\
\text { - } & \text { Medios de comunicación } \\
\text { - } & \text { Educación pública }\end{array}$ \\
\hline El plano internacional & $\begin{array}{l}\text { - Consecuencias para la salud de los acuerdos comerciales Inter- } \\
\text { nacionales } \\
\text { - } \\
\text { - } \\
\text { - } \\
\text { - Inuda externa a los programas de medicamentos esenciales }\end{array}$ \\
\hline
\end{tabular}

Fuente: 5 (p. 20)

y Social en el que se consigna la Política Farmacéutica Nacional como un paso en el camino correcto, que proporciona un marco de acción, acompañado de la asignación de recursos y de un sistema de seguimiento y evaluación, que puede constituir el espacio social y legal para que se valore la importancia del sector farmacéutico en la reorganización del sistema de salud y se aporten soluciones a un problema global. (6, p. 129)

El conjunto de actores convocados debe arrojar los criterios básicos y posibles, sobre los cuales atender la definición de la PFN y de la PFT. En este sentido, se espera que no solo coincidan en sus intereses individuales, sino que además establezcan los compromisos que asumirían a la hora de ponerse en marcha la política pública. Ello implica responder a los diferentes sectores relacionados y con los cuales tienen divergencias con respecto al tema.

La política pública tanto nacional como territorial haría posible que los convocados compartieran intereses y que se pusieran de acuerdo en las diferencias. Dicho escenario requiere un aporte apreciable de recursos 
Tabla 2. Farmacovigilancia

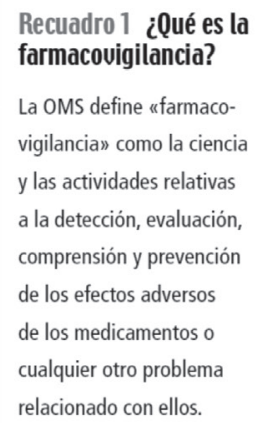

\section{Figura 1 Desarrollo clínico de los medicamentos}

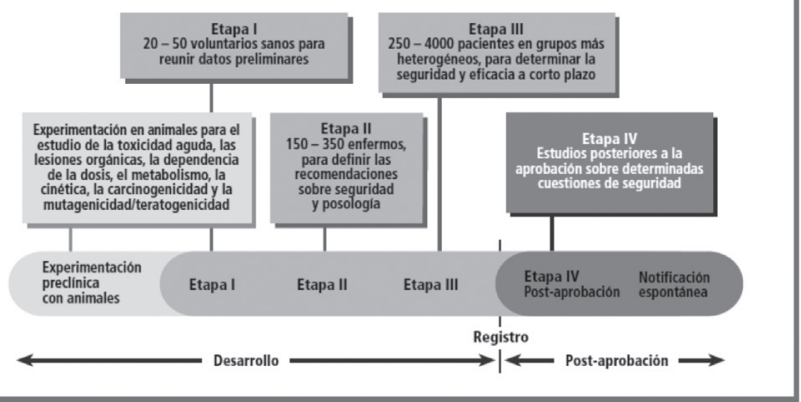

Fuente: 7 (p. 9)

por cuenta de los organismos internacionales, de los propios gobiernos y también de los actores recurrentes en el sector. Por ende, se espera que temas como la farmacovigilancia (ver tabla 2) sean parte de los elementos de trabajo que es necesario considerar por parte de ellos:

El término Farmacoepidemiología se refiere al estudio del uso y los efectos de los fármacos en grandes poblaciones. Otros autores lo definen como "la ciencia que estudia el impacto de los fármacos en poblaciones humanas utilizando para ello el método epidemiológico" o "la aplicación del conocimiento, método y razonamiento epidemiológicos al estudio de los efectos (beneficios y adversos) y usos de los fármacos en poblaciones humanas". (8, p. 129)

La PFN y la PFN superan las pretensiones establecidas en el Conpes 155, entre otras razones, por la naturaleza misma del sector de medicamentos, que a pesar de su regulación presenta comportamientos erráticos que incluso no alcanzan a medirse a través del mercado. El éxito de ambas políticas pasa por el concurso e interés que atraigan los diversos convocados para que estas tengan sentido, en cuanto al mercado, pero también dentro las condiciones políticas y sociales (tabla 3 ).
La PFN y la PFT deben contar, en primer lugar, con la guía de los principales organismos relacionados en la materia, de cara a un diálogo directo con las empresas y gremios dedicados a la producción de medicamentos a gran escala. Segundo, con la definición de un marco de política pública orientado por los estamentos relevantes, como el Ministerio de Salud y Protección Social, además de las secretarías de salud departamental, para que la política farmacéutica ciertamente tenga una clara orientación y metas a escala nacional y territorial.

\section{Análisis metodológico de la política farmacéutica nacional colombiana con respecto a la definición de la política pública farmacéutica territorial}

La metodología marco lógico, para el efecto, responde de manera idónea para ejemplarizar lo que implica la materialización de una política pública farmacéutica a escala nacional. La eficiencia de la política pública radica en ofrecer elementos con base en los cuales proponer programas, proyectos y subprogramas con los que hacer transferible la política pública nacional. Así, los planes de 


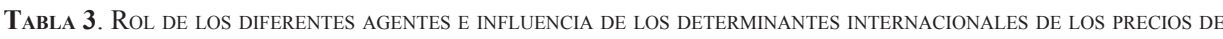
LOS MEDICAMENTOS DE ALTO COSTO (MAC)

\begin{tabular}{|c|c|c|c|c|c|c|}
\hline Agente & Rol & Argumento & Interés & $\begin{array}{l}\text { Documentos } \\
\text { que sustentan } \\
\text { la posición }\end{array}$ & $\begin{array}{l}\text { Tensión y } \\
\text { conflicto }\end{array}$ & $\begin{array}{l}\text { Altemativa } \\
\text { a la situación } \\
\text { actual }\end{array}$ \\
\hline $\begin{array}{l}\text { Empresas } \\
\text { multinacionales }\end{array}$ & - Producir MAC & $\begin{array}{l}\text { - Justifican los } \\
\text { precios por } \\
\text { innovación y } \\
\text { costos de } \\
\text { investigación }\end{array}$ & $\begin{array}{l}\text { - Recuperar } \\
\text { costos } \\
\text { - Recibir estímulos } \\
\text { para futuras } \\
\text { investigaciones }\end{array}$ & $\begin{array}{l}\text { - ADPIC } \\
\text { - ADPIC plus }\end{array}$ & $\begin{array}{l}\text { - Con gobiemos } \\
\text { nacionales }\end{array}$ & $\begin{array}{l}\text { - Compatibilizar } \\
\text { intereses comerciales } \\
\text { con derechos } \\
\text { humanos y objetivos } \\
\text { de las políticas } \\
\text { sanitarias, asumiendo } \\
\text { responsabilidad social } \\
\text { para garantizar el } \\
\text { acceso a MAC } \\
\text { - Colaborar con los } \\
\text { gobiernos para } \\
\text { prevenir medidas que } \\
\text { afectarian sus } \\
\text { intereses económicos }\end{array}$ \\
\hline $\begin{array}{l}\text { Gobiemos } \\
\text { nacionales }\end{array}$ & $\begin{array}{l}\text { - Diseñar políticas } \\
\text { que garanticen el } \\
\text { cumplimiento de } \\
\text { los derechos } \\
\text { ciudadanos }\end{array}$ & $\begin{array}{l}\text { - Atienden las } \\
\text { necesidades de } \\
\text { la mayoría de la } \\
\text { población } \\
\text { - Administran bien } \\
\text { los fondos } \\
\text { públicos }\end{array}$ & $\begin{array}{l}\text { - Asegurar la } \\
\text { provisión de los } \\
\text { medicamentos y } \\
\text { tratamientos } \\
\text { necesarios, } \\
\text { cuidando que los } \\
\text { costos no } \\
\text { amenacen la } \\
\text { sostenibilidad } \\
\text { financiera del } \\
\text { pais }\end{array}$ & $\begin{array}{l}\text { - ADPIC } \\
\text { - Declaración } \\
\text { Universal de } \\
\text { DDHH } \\
\text { - Declaración de } \\
\text { Doha } \\
\text { - Agenda con la } \\
\text { OPS }\end{array}$ & $\begin{array}{l}\text { - Con empresas } \\
\text { multinacionales } \\
\text { - Con sus ciudadanos } \\
\text { en el caso de no } \\
\text { atender reclarmos } \\
\text { - Con el poder judicial } \\
\text { por verse forzados } \\
\text { a financiar al } \\
\text { judicializarse los } \\
\text { casos }\end{array}$ & $\begin{array}{l}\text { - Garantizar el acceso a } \\
\text { MAC y a la vez } \\
\text { sostener sus sistemas } \\
\text { financieros } \\
\text { - Cumplir con } \\
\text { los tratados } \\
\text { internacionales y } \\
\text { respetar la propiedad } \\
\text { intelectual } \\
\text { - Informarse sobre la } \\
\text { situación en otros } \\
\text { paises, buscar y } \\
\text { compartir buenas } \\
\text { prácticas } \\
\text { - Coordinar protocolos } \\
\text { de cobertura y } \\
\text { compras conjuntas }\end{array}$ \\
\hline $\begin{array}{l}\text { Aseguradoras } \\
\text { de salud }\end{array}$ & $\begin{array}{l}\text { - Cumplir con sus } \\
\text { obligaciones de } \\
\text { prestación de los } \\
\text { servicios a } \\
\text { afliados }\end{array}$ & $\begin{array}{l}\text { - Brindan } \\
\text { prestaciones } \\
\text { con equidad }\end{array}$ & $\begin{array}{l}\text { - Ofrecer buena } \\
\text { atención a sus } \\
\text { afiliados en caso } \\
\text { de enfermedad } \\
\text { - Recibir } \\
\text { ganancias con } \\
\text { los apostes de } \\
\text { sus asegurados }\end{array}$ & $\begin{array}{l}\text { - Declaración } \\
\text { Universal de } \\
\text { DDHH } \\
\text { - Legislación local }\end{array}$ & $\begin{array}{l}\text { - Con sus afiliados en } \\
\text { caso de no financiar } \\
\text { MAC }\end{array}$ & $\begin{array}{l}\text { - Cumplir sus } \\
\text { obligaciones con sus } \\
\text { asegurados } \\
\text { - Coordinar con los } \\
\text { gobiernos listados de } \\
\text { medicamentos obli- } \\
\text { gatorios, colaborando } \\
\text { en su elaboración con } \\
\text { criterios de costo- } \\
\text { eficiencia }\end{array}$ \\
\hline $\begin{array}{l}\text { Profesionales } \\
\text { de la salud }\end{array}$ & $\begin{array}{l}\text { - Cuidar la salud } \\
\text { de la población } \\
\text { - Recuperar la } \\
\text { salud } \\
\text { - Limitar el daño } \\
\text { - Reducir la } \\
\text { mortalidad }\end{array}$ & $\begin{array}{l}\text { - "Estamos para } \\
\text { atender a los } \\
\text { pacientes y no } \\
\text { para racionalizar } \\
\text { los gastos del } \\
\text { sistema" }\end{array}$ & $\begin{array}{l}\text { - Brindar a } \\
\text { sus pacientes } \\
\text { todas las } \\
\text { oportunidades } \\
\text { racionalmente } \\
\text { existentes para } \\
\text { el manejo de su } \\
\text { enfermedad }\end{array}$ & $\begin{array}{l}\text { - Declaración } \\
\text { Universal de } \\
\text { DDHH (art 17) }\end{array}$ & $\begin{array}{l}\text { - Con el gobierno en } \\
\text { el caso de prescribir } \\
\text { MAC en forma } \\
\text { irracional } \\
\text { - Con los ciudadanos } \\
\text { cuando no atienden } \\
\text { los reclamos de } \\
\text { prescripción de MAC } \\
\text { por sus pacientes }\end{array}$ & $\begin{array}{l}\text { - Ser conscientes al } \\
\text { prescribir, asegurando } \\
\text { la eficacia y costo- } \\
\text { eficiencia de lo que } \\
\text { medican } \\
\text { - Informarse sobre los } \\
\text { precios que hay en el } \\
\text { mercado y basarse en } \\
\text { evidencias a la hora de } \\
\text { tomar decisiones } \\
\text { clínicas y terapéuticas }\end{array}$ \\
\hline $\begin{array}{l}\text { Ciudadanos y } \\
\text { pacientes }\end{array}$ & $\begin{array}{l}\text { - Reclamar que se } \\
\text { garantice su } \\
\text { derecho al } \\
\text { acceso a la salud }\end{array}$ & $\begin{array}{l}\text { - "Tenemos } \\
\text { derecho a } \\
\text { acceder al mejor } \\
\text { tratamiento } \\
\text { disponible" }\end{array}$ & $\begin{array}{l}\text { - Acceder al } \\
\text { medicamento } \\
\text { que los } \\
\text { profesionales les } \\
\text { han prescripto } \\
\text { para tener la } \\
\text { oportunidad de } \\
\text { curarse }\end{array}$ & $\begin{array}{l}\text { - Declaración } \\
\text { Universal DDHH } \\
\text { (art 17) } \\
\text { - Convención } \\
\text { Americana de } \\
\text { DDHH }\end{array}$ & $\begin{array}{l}\text { - Con el gobierno o } \\
\text { con sus } \\
\text { aseguradoras si no } \\
\text { les proveen el } \\
\text { medicamento que } \\
\text { necesitan }\end{array}$ & $\begin{array}{l}\text { - Cooperar en la } \\
\text { racionalización del uso } \\
\text { de los medicamentos y } \\
\text { en la construcción de } \\
\text { una conciencia colectiva } \\
\text { sobre la importancia de } \\
\text { la eficacia clínica de un } \\
\text { fámaco } \\
\text { - Mantener la solidez de } \\
\text { los sistemas de salud }\end{array}$ \\
\hline Poder judicial & $\begin{array}{l}\text { - Asegurar que el } \\
\text { Estado cumpla } \\
\text { con sus } \\
\text { obligaciones, } \\
\text { velando por la } \\
\text { garantia real de } \\
\text { los derechos de } \\
\text { los ciudadanos }\end{array}$ & $\begin{array}{l}\text { - "Estamos del } \\
\text { lado de los } \\
\text { pacientes. } \\
\text { Ante la duda, } \\
\text { prevalece el } \\
\text { derecho humano } \\
\text { a preservar la } \\
\text { vida" }\end{array}$ & $\begin{array}{l}\text { - Cumplir con su } \\
\text { labor profesional }\end{array}$ & $\begin{array}{l}\text { - Declaración } \\
\text { Universal de } \\
\text { DDHH (art 17) } \\
\text { - Legislación } \\
\text { local }\end{array}$ & $\begin{array}{l}\text { - Con el gobiemo, al } \\
\text { cual se enfrenta } \\
\text { cuando se } \\
\text { judicializan los casos } \\
\text { de provisión de MAC } \\
\text { a los ciudadanos }\end{array}$ & $\begin{array}{l}\text { - Velar por el } \\
\text { cumplimiento de los } \\
\text { derechos humanos } \\
\text { - Tener amplia } \\
\text { conciencia de las } \\
\text { decisiones que toman, } \\
\text { basado en información } \\
\text { sólida y evidencia } \\
\text { cientifica } \\
\text { - Evaluar la relevancia } \\
\text { de sus decisiones } \\
\text { en el presupuesto } \\
\text { público, asegurando } \\
\text { racionalidad en los } \\
\text { gastos }\end{array}$ \\
\hline
\end{tabular}

Fuente: elaboración de los autores.
Nota: Abreviaturas. ADPIC: [Acuerdo de la Organización Mundial del Comercio] sobre "Aspectos de los derechos de propiedad intelectual relacionados con el comercio"; OPS: Organización Panamericana de la Salud, DDHH: Derechos humanos.

Fuente: 9 (pp. 170-1) 
desarrollo o la misma política farmacéutica deben cumplir con ello:

La Metodología de Marco Lógico es una herramienta para facilitar el proceso de conceptualización, diseño, ejecución y evaluación de proyectos. Su énfasis está centrado en la orientación por objetivos, la orientación hacia grupos beneficiarios y el facilitar la participación y la comunicación entre las partes interesadas. Puede utilizarse en todas las etapas del proyecto: En la identificación y valoración de actividades que encajen en el marco de los programas país, en la preparación del diseño de los proyectos de manera sistemática y lógica, en la valoración del diseño de los proyectos, en la implementación de los proyectos aprobados y en el Monitoreo, revisión y evaluación del progreso y desempeño de los proyectos (The Logical Framework Approach. AusguidElines, Ausaid). (10, p. 2)

El marco lógico está concebido para que las políticas en cualquier terreno pasen a revisión colectiva y social. La trascendencia de dicho recorrido radica en que la propia población reconoce su participación a la hora de pasar revista a los cometidos o balances generados en los temas considerados desde el marco lógico. De allí que su alcance trascienda la observación de los grupos de interés o presión que pudiesen entorpecer o restringir el propósito de los parámetros de la iniciativa:

Para la inclusión del marco lógico en la evaluación de políticas de ejecución descentralizada, es necesario un nivel de institucionalización presente en los tres niveles de gobierno involucrados. Ello además de elementos estructurales y de recursos, requiere de comportamientos corresponsables entre los involucrados y que los evaluadores puedan aplicar su conocimiento y experiencia para identificar y expresar problemáticas y diseñar soluciones. (11, p. 77)

La metodología parte del criterio holístico de participación sobre el que diferentes agentes o actores, al igual que estamentos y grupos, hacen parte del objeto o propósito referente a los temas que se tratan. El tomar posición o postura con relación a los planteamientos que allí se desarrollan, hace parte de las cualidades y condiciones sobre las que se recrea el ejercicio, sobre el que en últimas, lo más importante es la toma de decisiones de los agentes. El marco lógico pretende destacar el objeto del tema, más que una postura en particular (figura 1).

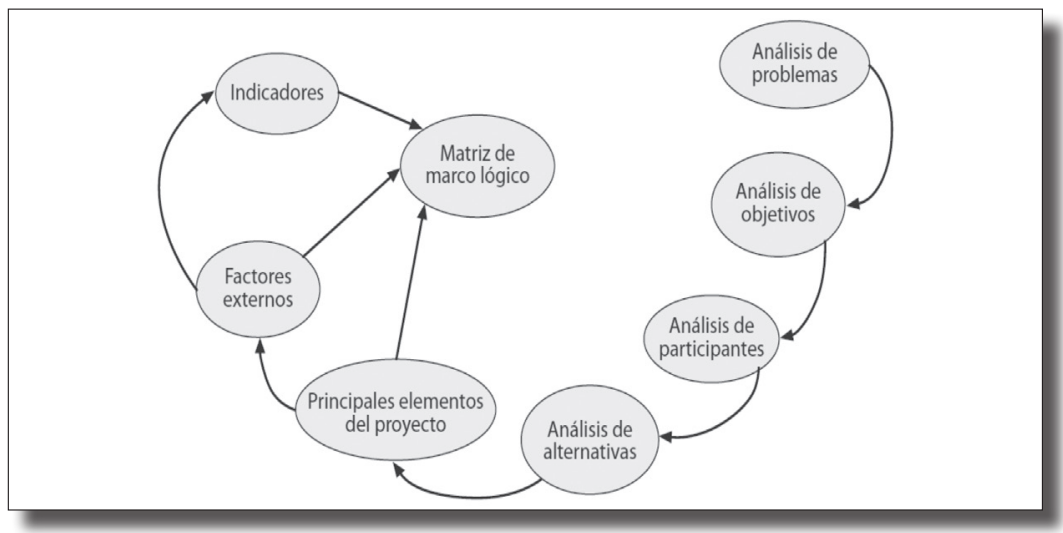

Figura 1. DinÁmica del EML EN TEORÍA

Fuente: 12 (p. 4) 


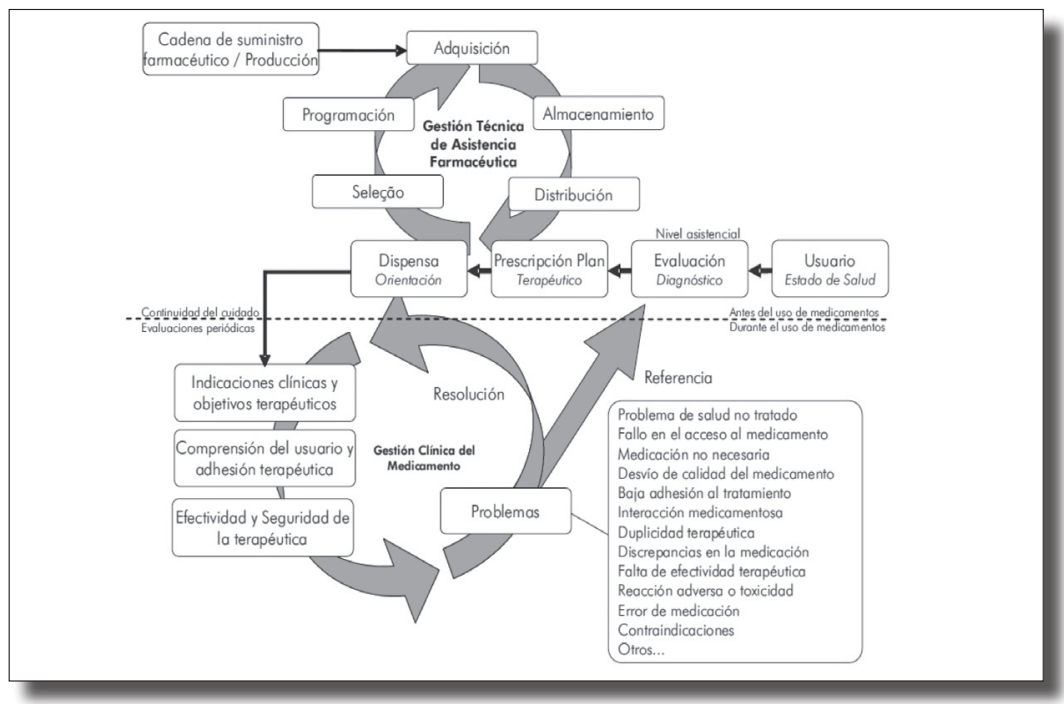

Figura 2. Modelo lógico-Conceptual de la af integrada al proceso de Cuidado en Salud Fuente: 13 (p. 44)

La interacción y vinculación del marco lógico como marco de referencia para alistar la política farmacéutica nacional y regional, permite de manera iterativa que los diferentes agentes y grupos definan o por lo menos planteen las razones o los elementos sobre los cuales debe abordarse alguna temática. Mientras más abierta y convocante sea la política, se entiende que tendrá más trascendencia y menos costos ocultos o aprovechamiento de intereses particulares (figura 2).

El marco lógico permite enfocar las diferentes intenciones previstas en el objeto temático, diagnóstico o problema en mención. Por ende, este tipo de metodología influye en los propósitos enfocados en la salud y, por supuesto, en los medicamentos. Sin embargo, son pocas las evidencias que expone el sector farmacéutico, agremiados, empresarial, poblacional y sindicado, a la hora de refrendar aspectos relacionados con la participación en los propósitos generales de este.

En Colombia, el sector farmacéutico es ajeno a la realidad del sistema de salud del país, siendo este un comportamiento común en sectores que tienen características oligopólicas, para los cuales el cierto margen del control en la ubicación en que se encuentran, les permite estar aislados de cualquier convergencia intersectorial. Mientras que cada sector planee sus propósitos por fuera de las condiciones y sostenibilidad del sistema, no habrá política nacional farmacéutica, hospitalaria o médica que tenga éxito:

$$
\begin{aligned}
& \text { La atención farmacéutica debería siempre } \\
& \text { estar contemplada dentro de la política na- } \\
& \text { cional de salud como parte de las acciones de } \\
& \text { prevención, promoción y recuperación consi- } \\
& \text { deradas en la atención sanitaria. Sin embar- } \\
& \text { go, la propia naturaleza del medicamento } \\
& \text { trasciende el aspecto sanitario, congregando } \\
& \text { otras esferas de la dinámica nacional, que lo } \\
& \text { convierten en un objeto diferenciado sobre } \\
& \text { el cual es necesario plantear el desarrollo de } \\
& \text { una política específica, de desarrollo cientí- } \\
& \text { fico y tecnológico. (14, p. 1037) }
\end{aligned}
$$

Si la PFN no tiene los mecanismos para transferir los preceptos de orden internacional, y a su vez tiene dificultades para ubicarse en el campo de lo regional y territorial, es mejor 


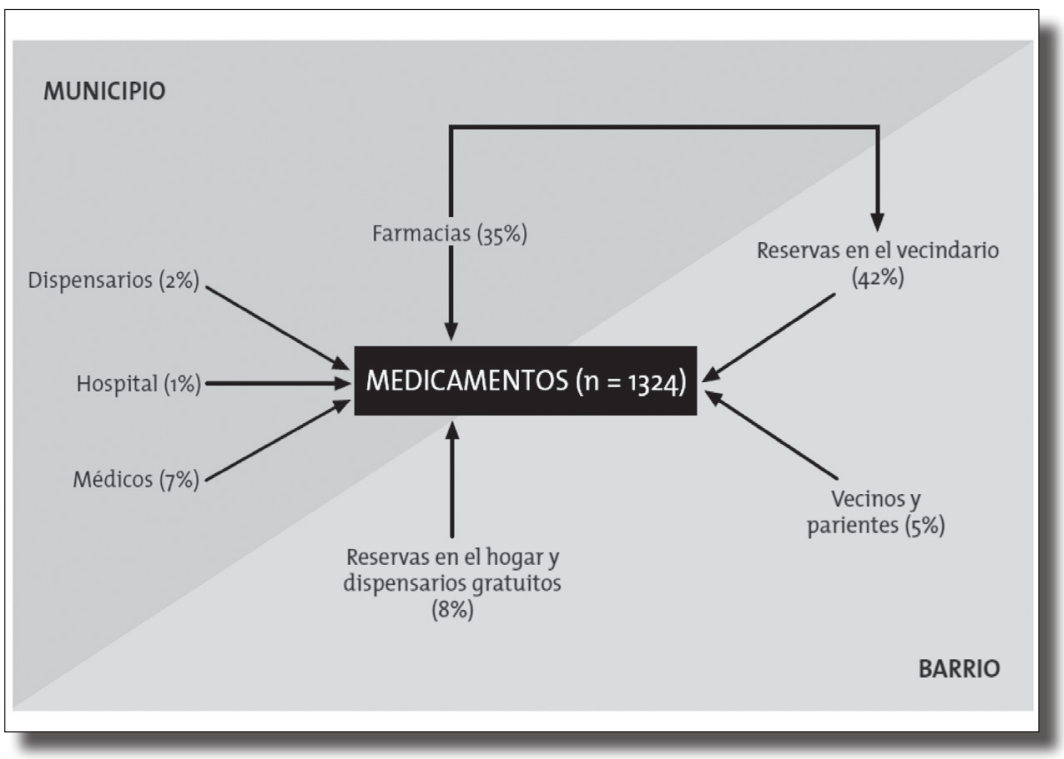

Figura 3. Canales de distribución de medicamentos en la comunidad: un ejemplo de Filipinas Fuente: 5 (p. 12)

pasar a la inseminación jurídica y legislativa, para que si bien no tenga recorrido de política, pueda advertirse en el marco de la norma. Es decir, el problema de la concepción de la política en cualquier caso pasa por su capacidad de asimilación, convocatoria y transferencia:

Los temas actualmente más relevantes y prevalentes cuando se trata del uso inadecuado de medicamentos incluyen polifarmacia, errores de medicación, no seguir directrices clínicas, discrepancias terapéuticas en la transición del usuario entre niveles asistenciales, baja efectividad de los tratamientos, ocurrencia de eventos adversos, automedicación irresponsable y baja adhesión a los tratamientos. En todo el mundo, crecen las evidencias del impacto de esos problemas sobre la población y sobre los sistemas de salud. (13, p. 42)

La incertidumbre en la que se encuentra inmerso el sistema de salud en Colombia y en el mundo deja poco margen para aventurar iniciativas como las consignadas en el Conpes
155 sobre PFN. En este sentido, queda solo para el despliegue de dicha iniciativa, que en cada departamento o región del país se comprenda la magnitud de lo que implica acoger disposiciones generales en materia de política pública, y cómo reconvertirlas en el marco de la trascendencia territorial:

Puntualizan la falta de información sobre el uso apropiado de los medicamentos, la concepción tergiversada del medicamento visto como un objeto de consumo cualquiera, y la práctica de automedicación. Finalmente, respecto a la entrega de medicamentos por los dispensadores, mencionan: el incumplimiento de las normas sobre venta de medicamentos (con receta, venta libre, bajo control): la falta de capacitación del personal responsable; el predominio del interés lucrativo sobre el comportamiento ético en la función de la dispensación, así como la falta de claridad sobre los parámetros para la sustitución de los medicamentos prescritos. (14, p. 1032) 
Es importante que el Ministerio de la Protección Social exija o plantee en los diferentes departamentos que se priorice el ejercicio de definición de política pública en materia de salud. No solamente es el escenario de los medicamentos, es toda la estructura que se requiere para que la salud, en toda su extensión, sea compatible con las condiciones que pregona el mercado. Es decir, que la política pública en salud se requiere para que, efectivamente, se logre el efecto esperado en dicho contexto, en el que el sector farmacéutico sume:

En resumen, hasta hoy parece que no se ha encontrado ningún método para la selección de medicamentos que resuelva o satisfaga los intereses sanitarios de los pacientes y cumpla con los objetivos económicos de los sistemas de salud que soportan los costes. $(15$, p. 10)

A pesar de ello, el sistema de salud pasa por alto el criterio de esencial, sobre el que debe priorizarse la dispensa del medicamento. No obstante, el abundante contenido generado a través de los mecanismos de acceso, considerados filtros en su momento, ha terminado por impedir que haya claridad en materia de medicamentos. Por ende, la política nacional o territorial que se formule en cualquiera de ellos debe corresponder a una evaluación territorial:

En Colombia existe un seguro de salud obligatorio para la población. El paquete de beneficios, denominado Plan Obligatorio de Salud (POS), contempla el acceso a medicamentos esenciales para los ámbitos hospitalario y ambulatorio. El contenido del pos es diferenciado según el régimen de afiliación (contributivo y subsidiado). Desde el 2008 mediante la expedición de la Sentencia T760 de la Corte Constitucional, el país adelanta esfuerzos para la unificación del plan de beneficios de ambos regímenes. (16, p. 40)
El sector farmacéutico, como el resto del sistema de salud en Colombia, se encuentra capturado públicamente y en cuidados intensivos. El otro escenario, es el grado de respuesta que puede tener el sistema para permitir el acceso o la incorporación ad hoc de propuestas en materia de política farmacéutica. Es claro que la propuesta, como el sistema de salud, carece de filtros o de reciprocidad, en donde las respuestas también tengan consideración y de paso, respuesta.

\section{Definición de la política pública farmacéutica regional y territorial en Colombia}

La metodología parte de la premisa relacionada con la convocatoria de la población a la hora de analizar los temas en general. Después de ello se establecen los problemas más apremiantes, que son evaluados para identificar un problema central que recoja los expuestos inicialmente. A partir de allí, se plantean diversos objetivos a través de los cuales se determina el central. Luego se plantean las posibles alternativas de selección que seguir como parte de las propuestas para avanzar. Posteriormente, se plantea una matriz de planificación de proyectos, que luego de aprobarse terminan insertos en la matriz lógica de planeación:

\footnotetext{
El seguimiento del tratamiento farmacológico debe considerar las siguientes situaciones: a) la recolección de la información no está limitada a la entrevista con el paciente, ya que se dispone de documentación escrita (hojas de prescripción, hojas de enfermería, historias clínicas, etc.) e información oral de diferentes miembros del equipo de salud: $b$ ) la comunicación de las intervenciones farmacéuticas debe ser ágil y no sólo contemplar al prescriptor sino a todo el equipo de salud, especialmente a enfermería; b) las activida-
} 
des de educación sanitaria no se limitan al paciente individual, pudiendo incorporar a los profesionales de la institución; c) al momento del alta, el farmacéutico debe generar información Farmacoterapéutica tanto para el paciente como para los profesionales que lo atiendan en forma ambulatoria (incluyendo también al farmacéutico comunitario). (17, p. 85)

La convocatoria a los diferentes actores del sistema salud representa el primer criterio al que debe atender el marco lógico. En igualdad de condiciones y posibilidades, cada uno expone lo que realmente sucede en materia de salud, en donde se encuentra la dispensación de medicamentos. En este frente se identifica, por parte de todos, el problema central que finalmente debe atenderse. Así que es necesario el reconocimiento de todos los participantes, incluyendo los contrarios, que podrían ser quienes tienen capturado al sistema (tabla 4).

La tabla anterior indica, de manera proactiva, los aspectos centrales que es necesario contemplar para los convocados en el proceso de elaboración de la política pública, conforme al derrotero del marco lógico. En este ejercicio, los llamados a participar serían las asociaciones de pacientes, en calidad de beneficiados y las familias, a la par que las asociaciones clínicas, hospitalarias y médicas en calidad de beneficiarios indirectos. A su vez, el conjunto de los neutros estaría conformado por el grueso de entidades públicas vigilantes de la salud. Asimismo, en los perjudicados podrían estar las firmas de laboratorios y las empresas (tabla 5).

La identificación del problema central pasa por la observancia de todos los convocados, quienes revisan uno a uno los temas planteados como problemas, sacando a flote el que más contenga o represente el conjunto de los problemas analizados. De allí que el identificado como problema central aglutine las expectativas de todos los convocados, siendo esta la primera ventaja que en esencia arroja la metodología de marco lógico.

El tránsito que debe cursar el medicamento, en versión genérica o de laboratorio, es el que costos de transacción que debe agregar el paciente para que el ciclo del programa o paquete de salud sea completado. Así, la cita médica, la

TABla 4. Ubicación DE ACTORES - NiVELES y VARIABLES

\begin{tabular}{|c|c|}
\hline Nivel & Variables \\
\hline Prescripción & $\begin{array}{l}\text { Fármaco, precio,dosis, número de unidades, adecuación al protocolo (si/) } \\
\text { no), antes o después de los precios de referencia. }\end{array}$ \\
\hline Pacientes & $\begin{array}{l}\text { Edad, género, activo o pensionista, número de episodios, consumo de } \\
\text { recursos o utilización total en el año, episodio psiquiátrico, duración del } \\
\text { tratamiento }\end{array}$ \\
\hline Médicos & $\begin{array}{l}\text { Edad, género, años de experiencia, nivel educativo, años en el puesto, } \\
\text { número de pacientes a su cargo, porcentaje de pacientes activos y pensio- } \\
\text { nistas, presión asistencial (número medio de pacientes atendidos al día) y } \\
\text { perfil de la prescripción (proporción de prescripciones por paciente o visita) }\end{array}$ \\
\hline Centros de Salud & Número de médicos y perfil, población adscrita, rural o urbano \\
\hline $\begin{array}{l}\text { Zonificación (especialista } \\
\text { de referencia) }\end{array}$ & $\begin{array}{l}\text { Indicadores del entorno socioeconómico; efecto inducción por el espe- } \\
\text { cialista }\end{array}$ \\
\hline
\end{tabular}

Fuente: 18 (p. 179) 
Tabla 5. Lista de convocados Pfd

\begin{tabular}{|c|c|c|}
\hline Convocados & Diagnóstico & Problema(s) \\
\hline $\begin{array}{l}\text { Beneficiarios: } \\
\text { - } \quad \text { Empresas Prestadoras de } \\
\text { Salud (EPS) } \\
\text { - Laboratorios biotecnoló- } \\
\text { gicos } \\
\text { - Empresas genéricas }\end{array}$ & $\begin{array}{l}\text { El sistema de salud les resulta } \\
\text { benigno, por cuanto es el } \\
\text { único sector del sistema que } \\
\text { se encuentra fortalecido con } \\
\text { el actual esquema de salud } \\
\text { del país }\end{array}$ & $\begin{array}{l}\text { La imposibilidad de acceder a } \\
\text { los medicamentos en cualquier } \\
\text { nivel }\end{array}$ \\
\hline $\begin{array}{l}\text { Contrarios: } \\
\text { - } \quad \text { Asociaciones de Pacientes } \\
\text { - } \text { Asociaciones médicas } \\
\text { - } \quad \text { Gremios de la Salud }\end{array}$ & $\begin{array}{l}\text { Es difícil identificar quién está } \\
\text { en contra del funcionamiento } \\
\text { del sistema de salud y la políti- } \\
\text { ca farmacéutica. No obstante, } \\
\text { las discrepancias los ubican en } \\
\text { dicho frente }\end{array}$ & $\begin{array}{l}\text { Es intrascendente el organi- } \\
\text { grama creado para representar } \\
\text { los intereses del sector de la } \\
\text { salud }\end{array}$ \\
\hline $\begin{array}{l}\text { Neutros: } \\
\text { - Instituto Nacional de Vigi- } \\
\text { lancia de Medicamentos y } \\
\text { Alimentos (Invima) } \\
\text { - Ministerio de Salud y la } \\
\text { Protección Social } \\
\text { - Superintendencia de Salud }\end{array}$ & $\begin{array}{l}\text { Escasamente cumplen la } \\
\text { función por la cual han sido } \\
\text { creados, en ningún momento } \\
\text { sobresalen por hacer que el } \\
\text { sistema de salud o el sector } \\
\text { farmacéutico funcionen }\end{array}$ & $\begin{array}{l}\text { Es poco reconocido el papel } \\
\text { que cumplen a la hora de } \\
\text { fomentar la salud como priori- } \\
\text { dad del Estado colombiano }\end{array}$ \\
\hline $\begin{array}{l}\text { Perjudicados: } \\
\text { - } \quad \text { Directos: pacientes } \\
\text { - Indirectos: familias } \\
\text { - Instituciones Prestadoras } \\
\text { de Salud (IPS) }\end{array}$ & $\begin{array}{l}\text { El alto costo de los dispositivos } \\
\text { médicos y los medicamentos }\end{array}$ & $\begin{array}{l}\text { La salud plantea condiciones } \\
\text { de rivalidad entre los diferen- } \\
\text { tes actores, hecho que hace } \\
\text { imposible generar políticas } \\
\text { públicas en cualquiera esfera }\end{array}$ \\
\hline
\end{tabular}

Fuente: elaboración propia

revisión y la prescripción, constituyen la hoja de ruta que debe realizar todo paciente o usuario en el sistema de salud. Particularmente, el último ciclo es el más difícil de alcanzar a la hora de evidenciar la eficiencia del sistema (figura 4).

El problema central, que para el caso puede ser establecido en cuanto a la fase de utili- zación del medicamento, debe generar los elementos para que se plantee el objetivo central de revertir dicha situación. Para ello, debe exponer los medios y los fines para que el objetivo sea alcanzable, medible y verificable, y que a su paso admita la posibilidad de que el sistema de salud genere impactos cualitativos y resultados cuantitativos (figura 5).

TABLA 6. LOS FaCtORES QUE INFLUYEN EN LA PRESCRIPCIÓN

\begin{tabular}{|l|l|}
\hline Tipo de influencia & Origen y sintomatología \\
\hline Externa & $\begin{array}{l}\text { Demanda del paciente: prescripciones de complacencia. } \\
\text { Demanda inducida por el especialista. } \\
\text { Presión de la industria e incentivos de marketing. } \\
-\quad \text { Fidelidad a la marca }\end{array}$ \\
\hline $\begin{array}{l}\text { Factores propios del } \\
\text { médico, moduladores } \\
\text { de conducta }\end{array}$ & $\begin{array}{l}\text { Presión asistencia. } \\
\text { Especialidad y formación de postgrado. } \\
\text { Sexo, edad, antigüedad, experiencia. }\end{array}$ \\
\hline
\end{tabular}




\begin{tabular}{|c|c|c|}
\hline Tipo de influencia & Origen y sintomatología & \\
\hline $\begin{array}{l}\text { Formación e informa- } \\
\text { ción. Intensidad y pro- } \\
\text { ductividad del esfuerzo } \\
\text { de busqueda }\end{array}$ & $\begin{array}{l}\text { Fuentes de información: } \\
\text { Experiencia propia. } \\
\text { - Volumen de práctica. } \\
\text { Red de colegas. } \\
\text { - Práctica aislada (me- } \\
\quad \text { dio rural). } \\
\text { Visitadores médicos, } \mathrm{Va} \text { - } \\
\text { demecum y esfuerzo de } \\
\text { laboratorios. } \\
\text { Guías farmacológicas. } \\
\text { Revistas de usar y tirar. } \\
\text { Revistas científicas }\end{array}$ & $\begin{array}{l}\text { La intensidad del esfuerzo de búsqueda } \\
\text { depende de: } \\
\text { - Volumen de práctica específico }(+) \text {. } \\
\text { - Volumen de práctica genérico }(-) \text {. } \\
\text { - Incertidumbre sustantiva sobre efectos } \\
\text { de tratamientos alternativos. } \\
\text { - Número de alternativas en el mercado. } \\
\text { La productividad del esfuerzo de búsqueda } \\
\text { depende de: } \\
\text { - Formación, experiencia y capacidad } \\
\text { (proxies: edad, antigüedad, credenciales } \\
\text { educativos). }\end{array}$ \\
\hline $\begin{array}{l}\text { Administración: regu- } \\
\text { lación, pautas, inter- } \\
\text { venciones, controles. }\end{array}$ & \multicolumn{2}{|c|}{$\begin{array}{l}\text { Regulación: Listas positivas y negativas, precios de referencia e incentivos a } \\
\text { la calidad de la prescripción y al control del gasto. } \\
\text { Controles directos (visados, ...). }\end{array}$} \\
\hline
\end{tabular}

Fuente: 18 (p. 186)

De no llegarse a un acuerdo relevante entre los actores convocados en la formulación de la PFD o regional, se considera necesario replantear todo el ejercicio a través de la apuesta del cuadro o marco de selección de alternativas. En dicho cuadro se plantean elementos no contemplados en el proceso anterior, para así identificar nuevas consideraciones o tópicos con los cuales recambiar el proceso inicial tomado por el diagnóstico precedente. La selección de alternativas, de manera colegiada, pretende identificar claramente el problema (figura 6).

La matriz de proyectos termina por orientar las posibles alternativas que se tienen para materializar las intenciones, pretensiones o

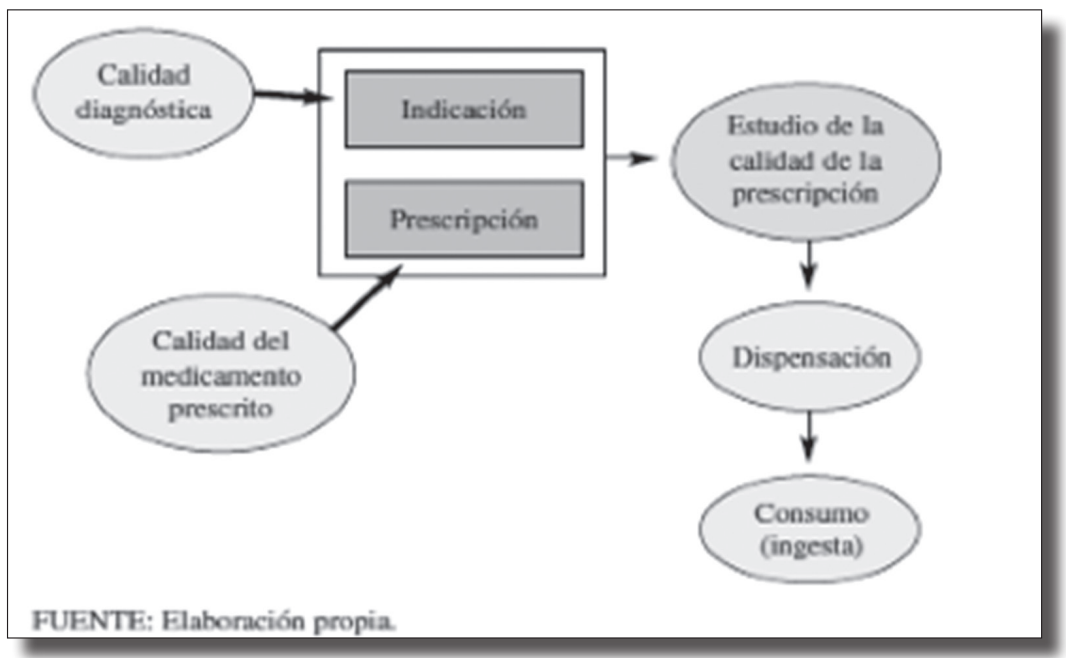

Figura 4. Fases de la utilización de LOS MEdicAmentos

Fuente: 18 (p. 163) 


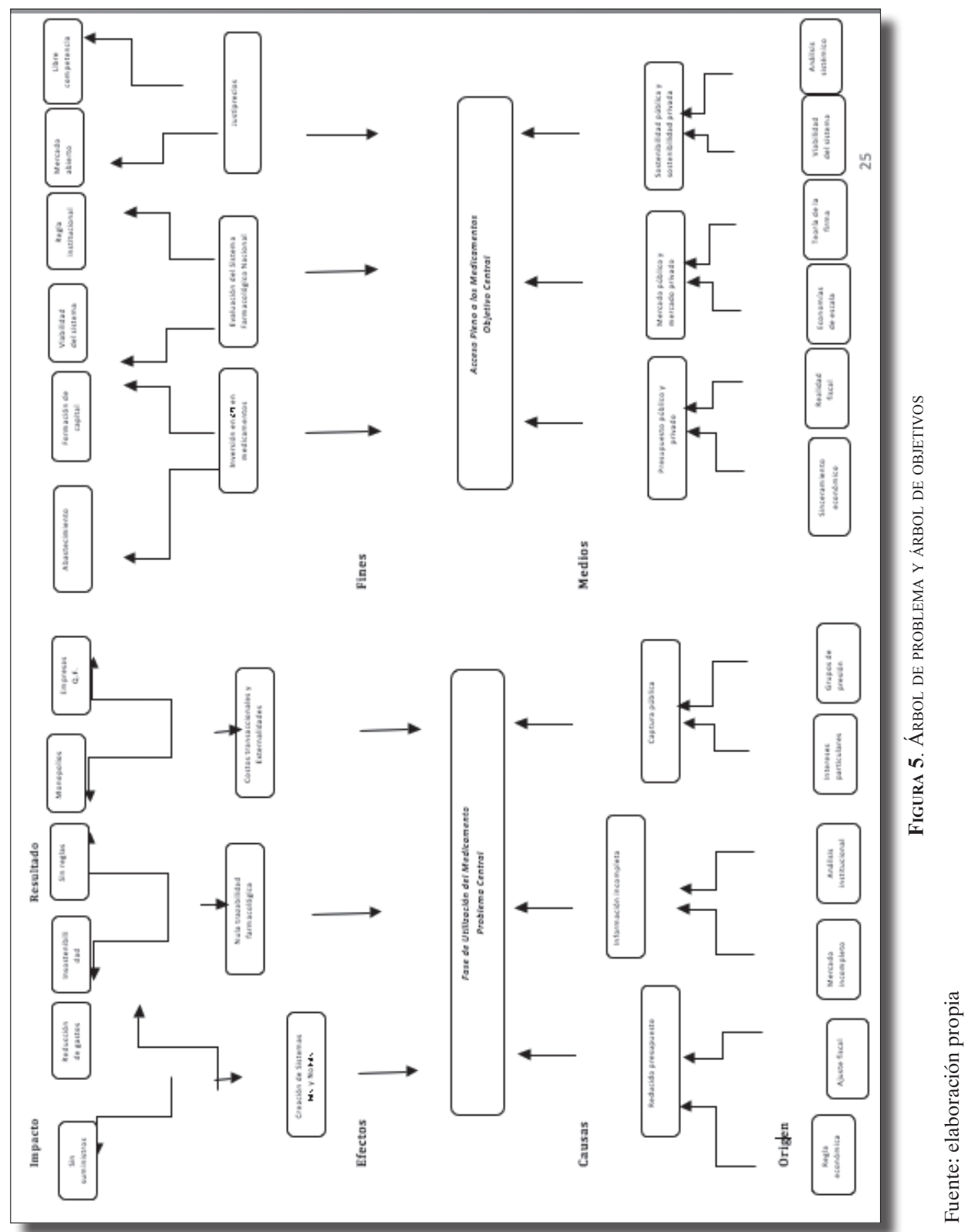




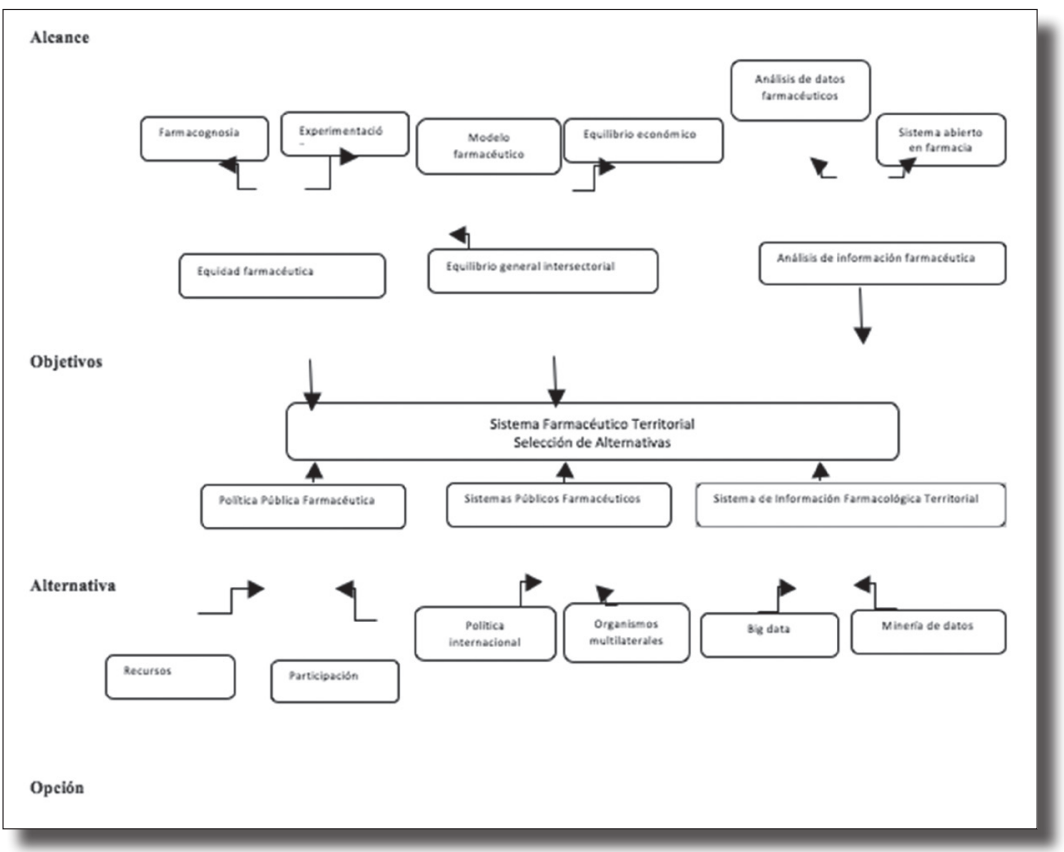

Figura 6. Selección de alternativas pft

Fuente: elaboración propia

resultados que se esperan de la adecuación de la PFT al sistema de salud en igual terreno, así como en el frente internacional. Por ende, su priorización corre por cuenta de la misma evaluación, a través de la cual participan los actores convocados inicialmente. Su despliegue pasa por la definición concreta de los proyectos que deben tomar camino para efectos de la política (tabla 7).

La ponderación de los proyectos pasa por el tamizaje de los convocados, quienes con suficiencia evalúan y valoran los resultados que pudiesen obtenerse con los proyectos. Seguidamente, estos al terminar siendo aprobados sobre cuantificaciones o mediciones preestablecidas, contribuyen para formular efectivamente la matriz de marco lógico delineada por el objetivo central. De allí la vinculación de todos los convocados pasa a considerarse desde la perspectiva técnica 56 prevista por la matriz de marco lógico, que recoge los fundamentos de los proyectos aprobados para incubarlos bajo los lineamientos de esta.

La matriz de marco lógico permite obtener un mejor análisis del alcance, las iniciativas, los proyectos y los resultados que podría arrojar la política farmacéutica, nacional e internacional. Por ende, la participación de cada uno de los convocados se revitaliza cuando se llega al propósito de ilustrar una real política en cualquier renglón privado o público. Al llegar a dicha cumbre, se espera que los proyectos o programas elaborados a través de la política pública, en este caso farmacéutica, tengan los resultados esperados por los convocados, escenario en el que se destaca la importancia de la participación hecha por todos.

\section{Conclusiones}




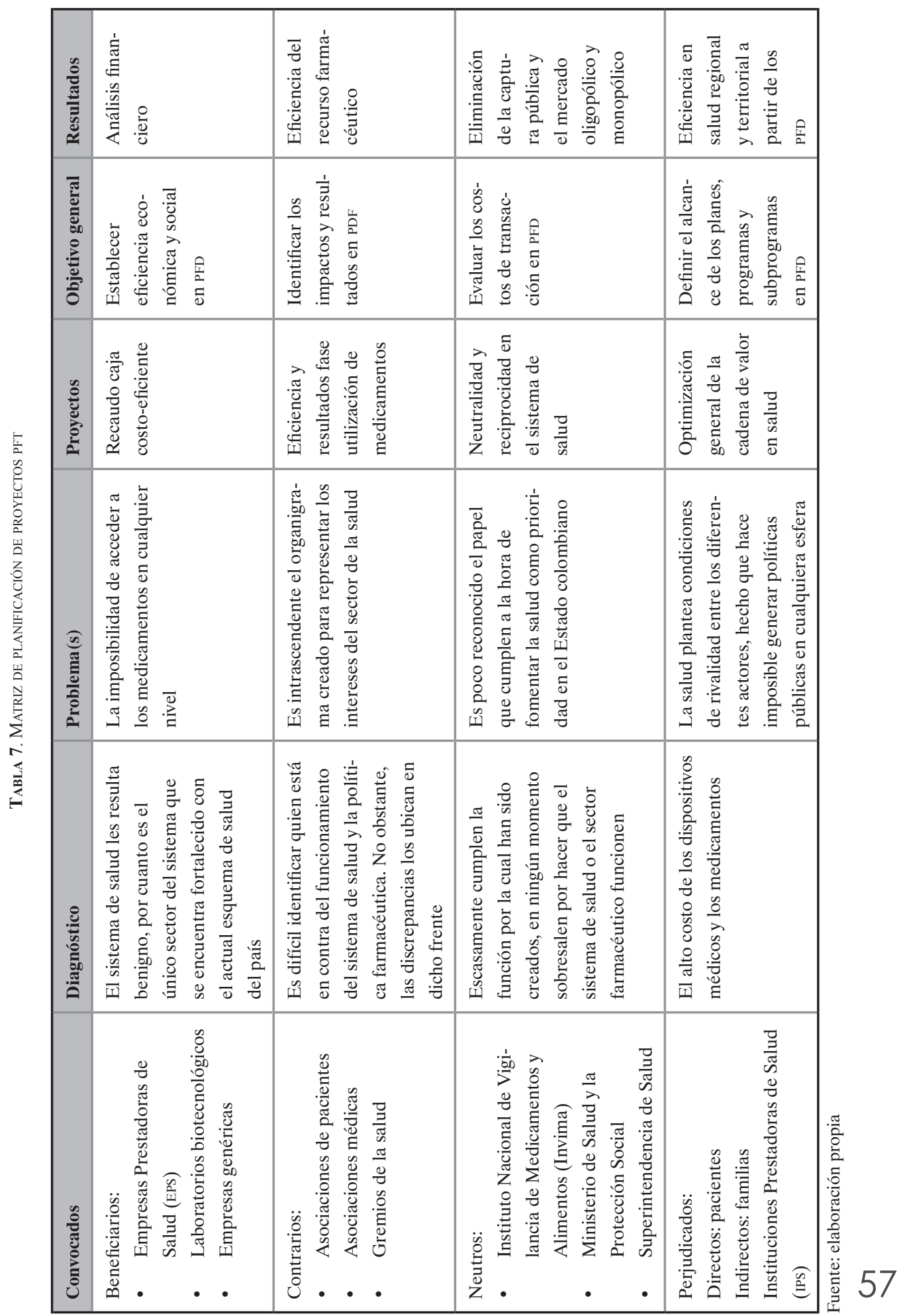


Tabla 8. Matriz de marco lóGico en PFT

\begin{tabular}{|c|c|c|c|c|}
\hline Meta & Indicadores & $\begin{array}{l}\text { Medios de verifi- } \\
\text { cación }\end{array}$ & $\begin{array}{l}\text { Supuestos o exter- } \\
\text { nalidades }\end{array}$ & \multirow{3}{*}{$\begin{array}{l}\text { Alcance y } \\
\text { evaluación de } \\
\text { objetivos }\end{array}$} \\
\hline $\begin{array}{l}\text { Propó- } \\
\text { sitos }\end{array}$ & $\begin{array}{l}\text { Establecer el sistema } \\
\text { de salud farmacéuti- } \\
\text { co nacional y territo- } \\
\text { rial en Colombia } \\
\text { Definir la viabilidad } \\
\text { compleja del sistema } \\
\text { de salud colombiano } \\
\text { con relación al sec- } \\
\text { tor farmacéutico } \\
\text { Proponer un modelo } \\
\text { intersectorial de } \\
\text { servicios de salud } \\
\text { territorial farmaco- } \\
\text { lógica }\end{array}$ & $\begin{array}{l}\text { Entidades multila- } \\
\text { terales y entidades } \\
\text { nacionales } \\
\text { Sistema de salud } \\
\text { internacional y } \\
\text { comportamiento } \\
\text { sectorial farmacéu- } \\
\text { tico } \\
\text { Política pública } \\
\text { nacional farmacéu- } \\
\text { tica y postulación de } \\
\text { la política territorial } \\
\text { farmacéutica }\end{array}$ & $\begin{array}{l}\text { Fallos (jurídicos) y } \\
\text { fallas (errores) del } \\
\text { sistema de salud } \\
\text { Grupos de presión } \\
\text { de multinacionales } \\
\text { y empresas del } \\
\text { sector químico } \\
\text { farmacéutico } \\
\text { Información incom- } \\
\text { pleta e idoneidad } \\
\text { de los recursos y } \\
\text { factores de trabajo } \\
\text { para el efecto }\end{array}$ & \\
\hline $\begin{array}{l}\text { Compo- } \\
\text { nentes }\end{array}$ & $\begin{array}{l}\text { Política nacional } \\
\text { farmacéutica } \\
\text { Política territorial } \\
\text { farmacéutica } \\
\text { Política local farma- } \\
\text { céutica } \\
\text { Planes de desarrollo } \\
\text { departamental }\end{array}$ & $\begin{array}{l}\text { Indicadores de } \\
\text { salud internacional } \\
\text { Datos e indicadores } \\
\text { de salud territorial } \\
\text { Impactos y resulta- } \\
\text { dos en salud } \\
\text { Secretaria de salud } \\
\text { departamental }\end{array}$ & $\begin{array}{l}\text { Fuentes de infor- } \\
\text { mación } \\
\text { Financiación y } \\
\text { viabilidad } \\
\text { Trazabilidad en } \\
\text { salud }\end{array}$ & \\
\hline $\begin{array}{l}\text { Activi- } \\
\text { dades }\end{array}$ & $\begin{array}{l}\text { Vinculación de pro- } \\
\text { gramas académicos } \\
\text { en Q F en la política } \\
\text { pública farmacéutica } \\
\text { Capacitación de } \\
\text { personal asistencial } \\
\text { en droguerías y } \\
\text { farmacias }\end{array}$ & $\begin{array}{l}\text { Análisis indicado- } \\
\text { res salud pública } \\
\text { territorial } \\
\text { Recursos para la ge- } \\
\text { neración de progra- } \\
\text { mas de intervención } \\
\text { farmacológica } \\
\text { Educación en pres- } \\
\text { cripción farmacéuti- } \\
\text { ca poblacional }\end{array}$ & $\begin{array}{l}\text { El manejo de políti- } \\
\text { ca pública } \\
\text { La sincronía de los } \\
\text { agentes, empresas y } \\
\text { grupos de interés } \\
\text { Resultados e impac- } \\
\text { tos en la población }\end{array}$ & $\begin{array}{l}\text { Programación } \\
\text { internacional } \\
\text { en PFN }\end{array}$ \\
\hline & & $\begin{array}{l}\text { Conforme a los re- } \\
\text { sultados obtenidos }\end{array}$ & $\begin{array}{l}\text { Conforme a los im- } \\
\text { pactos generados }\end{array}$ & $\begin{array}{l}\text { Programación } \\
\text { nacional y terri- } \\
\text { torial en PFT }\end{array}$ \\
\hline & & \multicolumn{3}{|c|}{ Evaluación económica y financiera } \\
\hline
\end{tabular}


Las directrices que plantean la OMs, la ops y los organismos adscritos no logran materializar una política internacional en materia de fármacos y medicamentos, como también sucede con otros frentes del sistema de salud. No obstante, la disposición de las patentes, luego de obtener el máximo beneficio, por parte de las empresas de genéricos, constituye el principal logro del sistema a escala mundial.

El proceso de formulación de la política pública farmacéutica en Colombia requiere un desarrollo amplio de la línea de trabajo institucional (económica jurídica), a través de la que puedan recogerse evidencias de trabajo relacionadas con el alcance, los propósitos, las metas y los resultados logrados con las disposiciones amparadas en la formulación de la política pública.

El éxito de la PFN pasa por la capacidad de filtro e injerencia que tenga con respecto a las estrategias de política farmacéutica territorial en cualquier terreno. La PFT permite precisamente orientar los principios de la OMS y la PFN hacia el desarrollo de mecanismos de gestión sobre los que efectivamente se atiendan los requerimientos en materia farmacéutica.

\section{Referencias bibliográficas}

1. Consejo Nacional de Política Económica y Social República de Colombia, Departamento Nacional de Planeación. Documento Conpes Social 155. Política Farmacéutica Nacional. Bogotá: 2012.

2. Pérez J. Marco lógico para la selección de medicamentos. Rev Cubana Med Gen Integr. 2000; 16 (2): 177-85.

3. Ministerio de la Protección Social, Organización Panamericana de la Salud (ops). Monitoreo de la situación farmacéutica en Colombia. Bogotá: Editorial Carrera 7a. 2003.

4. Collazo M, Sosa I. La farmacoeconomía: ¿Debe ser de interés para evaluar la eficiencia en la toma de decisiones? Rev Colomb Cienc Quim Farm. 2011; 40 (1): 54-66.

5. Hardon A, Hodgkin C, Fresle D. ¿Cómo investigar el uso de medicamentos por parte de los consumidores? Comunidad Europea, Organización Mundial de la Salud, Universidad de Ámsterdam; 2004.
6. Encuentro Nacional de Académicos de la Salud. Editorial, Ante la crisis del sistema de salud la academia tiene la palabra. Pronunciamiento de las instituciones académicas y de los centros de investigación de la salud sobre la necesidad de una reforma. Revista Facultad Nacional de Salud Pública Universidad de Antioquia. 2012; 30 (2): 127-30.

7. Organización Mundial de la Salud. La farmacovigilancia: garantía de seguridad en el uso de los medicamentos. Perspectivas Políticas de la oms sobre medicamentos. Ginebra: oms; 2004. pp. 9-15.

8. Álvarez F. Farmacoepidemiología. Estudios de utilización de medicamentos. Parte I: Concepto y metodología. Seguim Farmacoter. 2004. 2 (3): 129-36.

9. Marín GH, Polach MA. Medicamentos de alto costo: analisis y propuestas para los países del Mercosur. Rev Panam Salud Pública. 2011: 30 (2): 167-76.

10. Ortegón E, Pacheco JF, Prieto A. Metodología del marco lógico para la planificación, el seguimiento y la evaluación de proyectos y programas. Instituto Latinoamericano y del Caribe de Planificación Económica y Social (Ilpes), Área de Proyectos y Programas de Inversiones. Serie Manuales; 2005. pp. 1-86.

11. Cruz M. Análisis del marco lógico en la evaluación de programas sociales. Caso: programa 3 x 1 para migrantes [tesis de maestría en Gobierno y Asuntos Públicos]. COnAcyT - México, Flacso; 2008.

12. Medina H. Diseño de proyectos de inversión con el enfoque de marco lógico. San José de Costa Rica: IICA; 2009.

13. Januario C, Soler O, Fleith M. Servicios farmacéuticos integrados al proceso de cuidado en salud: gestión clínica del medicamento. Rev Pan Amoz Saude. 2011; 2 (3): 41-9.

14. Mejía S, Vélez AL, Buritica OC, Del Río JA. La política farmacéutica nacional en Colombia y la reforma de la seguridad social: acceso y uso racional de medicamentos. Cad Saude Pública; 2002: 18 (4): 1025-39.

15. Jiménez NV, Climente MM, Colomer J, Pérez C. Metodologías para la selección de medicamentos en el Hospital. Farm Hosp. Universidad de Valencia; 2000. 24 (1): 1-11.

16. Huertas VNR. Perfil farmacéutico de la República de Colombia. Ministerio de Salud y Protección Social y Organización Panamericana de la Salud/ Organización Mundial de la Salud (ops/OMs); 2012.

17. Fontana D, Uema S, Solá N. Seguimiento Farmacoterapéutico en el ámbito hospitalario: Análisis DAFO. Acta Farm Bonaerense. 2003; 22 (1): 81-6.

18. Gonzáles BC, Cabeza A, López A, Díaz JA, Álamo F, Ortún V. Evolución de los estudios de utilización de medicamentos: del consumo a la calidad de la prescripción. Cuadernos Económicos -ICE- (67): 161-89. 